\title{
Airway management and anesthesia for airway surgery: a narrative review
}

\author{
Lucy T. Li^, Hovig V. Chitilian, Paul H. Alfille, Xiaodong Bao \\ Department of Anesthesia, Critical Care and Pain Medicine, Massachusetts General Hospital, Boston, MA, USA \\ Contributions: (I) Conception and design: LT Li, X Bao; (II) Administrative support: None; (III) Provision of study materials or patients: None; (IV) \\ Collection and assembly of data: None; (V) Data analysis and interpretation: None; (VI) Manuscript writing: All authors; (VII) Final approval of \\ manuscript: All authors. \\ Correspondence to: Xiaodong Bao, MD PhD. Department of Anesthesia, Critical Care and Pain Medicine, Massachusetts General Hospital, GRB-444, \\ 55 Fruit Street, Boston, MA, USA. Email: xbao1@mgh.harvard.edu.
}

Objective: To discuss and summarize the literature for airway and anesthetic management tools the
anesthesiologist can use for airway surgery to both successfully manage the patient's physiological needs and
provide the surgeon the optimal surgical conditions with which to perform the surgery safely.
Background: The airway and anesthetic management of patients presenting for thoracic surgery poses
the anesthesiologist with a unique set of challenges, but also a unique set of opportunities to artfully utilize
and adapt a variety of management options that has developed over several decades of innovation. Sixty
years ago, airway surgery was initially performed with the patient spontaneously breathing and providing
anesthesia with halogenated agents and airway topicalization. As medicine entered the latter half of the
twentieth century with its development of new airway devices and modern anesthetic agents, most airway
surgeries could be safely performed under general anesthesia with secured airways. Today, with continued
technological advancements in surgical techniques and an expanding population of challenging patients, the
application of nonintubated anesthetic techniques and extracorporeal support is on the rise.

Methods: We conduct a narrative review of the literature on the history of airway and anesthetic management for thoracic surgery, the current management methods and evidence for each modality, and discuss future directions for the field.

Conclusions: While the airway and anesthetic management for airway surgery is challenging, the anesthesiologist has a variety of options including cross-field ventilation, jet ventilation, nonintubated techniques, and extracorporeal support to safely care for the patient. Whichever methods are chosen for the patient and surgery, thoracic surgery remains uniquely positioned in its need for close sharing and collaboration of all airway and anesthetic management decisions between the anesthesiologist and the surgeon.

Keywords: Anesthesia; jet; nonintubated; extracorporeal membrane oxygenation (ECMO)

Submitted Oct 29, 2021. Accepted for publication Dec 16, 2021.

doi: $10.21037 /$ tlcr-21-917

View this article at: https://dx.doi.org/10.21037/tlcr-21-917

\section{Introduction}

Airway surgery presents a unique situation in anesthesia where the anesthesiologist must share the airway in a highly coordinated fashion with the surgeon. Anesthesiologists must balance two major goals: minimizing obstruction of the surgical field while maintaining oxygenation and ventilation of the patient. More importantly, the anesthetic plan must be tailored to meet the challenges of managing

^ ORCID: 0000-0002-9090-0074. 
a critical airway preoperatively, a disrupted airway intraoperatively, and a tenuous airway postoperatively.

Half a century ago, prior to the advent of doublelumen tubes (DLTs) and modern anesthetic agents, many airway surgeries were performed with topical anesthesia and patients spontaneously breathing inhalational agents for fear of losing the airway. With the advancement of surgical techniques and seminal works by Hermes Grillo and other pioneers, most airway surgery nowadays can be safely performed under general anesthesia with a secured airway and muscle relaxation which provide a stable and quiet surgical environment. Depending on the nature of the lesion, a variety of airway and anesthetic management options have been developed ranging from cross-field ventilation to endobronchial intubation to jet ventilation. With the rapid progress of minimally invasive and robotic techniques during the past decade, there has been a rekindled interest in performing operations under nonintubated techniques to avoid instrumenting airways. Accumulation of experience with extracorporeal support has further opened a new avenue to perform surgery in previously inoperable patients.

This article will present a comprehensive and in-depth review of the available literature and evidence for all of the aforementioned different airway techniques and anesthetic methods and their applications for a variety of airway operations. This narrative overview particularly emphasizes the most recent developments of airway management from non-intubated techniques to extracorporeal support. Due to the heterogeneity of patients and local expertise, it is imprudent to make recommendations of airway management for every type of airway operation, and ultimately the choice of airway and anesthetic technique depends on the individual patient's comorbidities and the surgery to be undertaken. It bears remembering that no matter the methods chosen, it is always critical to maintain close communication with the surgeon and collaboratively determine the most appropriate approach for the patient and procedure.

We present the following article in accordance with the Narrative Review reporting checklist (available at https:// dx.doi.org/10.21037/tlcr-21-917).

\section{Methods}

We identified key published articles using the PubMed and Ovid databases and Google Scholar, published in English without time limitations up to October 2021. The search was conducted using combinations of terms: airway surgery, tracheal surgery, carinal surgery, nonintubated thoracic surgery, jet ventilation, and extracorporeal membrane oxygenation. We also performed secondary review of bibliographies of several key meta-analyses.

\section{Cross-field ventilation}

The easiest way to maintain ventilation in patients with a disrupted airway is to re-establish an airway via an endotracheal or endobronchial tube that is inserted into the distal airway by surgeons under direct visualization. This technique was initially described in an experimental model of tracheal surgery and later brought to clinical application in the 1950 s to 1960 s (1-3). The method was refined by Grillo et al. in tracheal surgery and quickly disseminated across the world (4). To this day, 93\% of peer-reviewed publications on tracheal surgery over the past half-century utilize cross-field ventilation with intermittent apnea for airway management (5).

Cross-field ventilation is simple to set up and does not require special equipment. The technique can be relatively easily adopted to provide a secure airway for most airway operations. The patient is intubated with an appropriately size endotracheal tube (ETT) in standard fashion by the anesthesiologist. Serial airway dilation by rigid bronchoscope or balloon may be required for airway stenosis to accommodate the ETT. Once the airway is opened by the surgeon, the orotracheal tube is withdrawn into the proximal airway, and a sterile endotracheal or endobronchial tube can be placed within the distal airway and is connected to sterile ventilation circuit. The circuit is passed from the surgical field to the anesthesia team and ventilation can be resumed. The operation can be carried out by working around the sterile endotracheal or endobronchial tube. Intermittent apnea may be necessary if the airway device needs to be removed for better surgical exposure. The technique requires a patient who can physiologically tolerate periods of apnea and extensive communication between the anesthesiologist and surgeon to coordinate the timing of ventilation versus apnea. During periods of ventilation, hyperventilation should be considered to support the apneic periods.

Once the reconstruction is almost complete and sutures are in place and ready to be tightened, the anesthesiologist assists the surgeon in advancing the orotracheal tube distal to the suture line and ventilation can resume orotracheally and the cross-field circuit is removed. The technique has 
proven to be invaluable in airway surgery (5). It provides secured positive-pressure ventilation and protection from aspiration. However, the surgical time can be prolonged due to obstruction of the surgical field by the airway device. Intermittent apnea can result in fluctuation of oxygen and carbon dioxide levels in patients. Certain airway surgeries may not be amenable to cross-field ventilation as it is contingent upon lesion size and location. Nevertheless, with appropriate patient selection and close communication between the surgical and anesthesia teams, cross-field ventilation has been utilized in over a thousand patients undergoing tracheal surgery at our center without issues (6).

\section{Jet ventilation}

In 1738, Daniel Bernoulli demonstrated the relationship of the pressure of a fluid with its speed, showing that with increased fluid speed, the pressure of the fluid decreased (7). The principle became the foundation for the early development of jet ventilation. In 1967, Douglas Sanders first described a new ventilation technique through the rigid bronchoscope in an open airway in which an activating toggle switched attached to an oxygen source delivered a jet of oxygen inside the lumen of a bronchoscope into the trachea via a variable pressure-reducing valve (8). In the late 1970s, Klain and Smith introduced high-frequency jet ventilation (HFJV) which was able to deliver jet flows at 60 to 100 cycles per minute (9).

In principle, jet ventilation applies a gas mixture under high pressure through a small-caliber, noncompliant catheter into the airway which is open to ambient air (10). Today, lowfrequency jet ventilation (LFJV) delivers jet flows at rate up to 20 cycles per minute, while HFJV performs at rate of 100 to 400 cycles per minute. Oxygenation and ventilation are determined by driving pressure, frequency, inspiratory time, and delivered fraction of inspired oxygen (10). During LFJV, air movement is conducted by convective flow from jet flow and air entrainment which leads to lung expansion and chest rise (11). The mechanism of HFJV is more complex and may involve Taylor dispersion, convective dispersion, molecular diffusion, cardiogenic mixing, pendelluft, and bulk flow (12).

Because jet ventilation does not mandate an airtight connection between the airway and ventilation, it holds unique advantages in airway surgery. Ventilation can be maintained when the airway is open during operations and both LFJV and HFJV have been applied in airway surgery. The small caliber catheter provides surgeons with better exposure and minimizes the need of repeated instrumentation of the distal airway $(5,13)$. The low mean airway pressure and small tidal volumes from HFJV result in minimal lung movement, providing a motionless lung that facilitates operations, in particularly for treating bronchopleural fistulas and tracheobronchial disruption. For distal airway surgery that requires one-lung ventilation (OLV), HFJV has been used to treat refractory hypoxemia during lung isolation when delivered to the nondependent operative lung through a DLT, bronchial blocker, or airway exchange catheter (14).

During jet ventilation, inspiration and insufflation are an active process via the jet of pulsed breaths and expiration is passive around the jet catheter tip. The intrinsic problem of jet ventilation is the risk of air trapping, auto-positive end-expiratory pressure, and barotrauma, which can be aggravated by airway stenosis $(15-17)$. This requires the anesthesia team carefully titrate parameters including inspiratory or driving pressure, frequency, and inspiration time. Upper airway obstruction interferes with the passive exhalation and increases the risk of barotrauma; thus, patients with morbid obesity and poor pulmonary compliance are not ideal candidates for LFJV. Other complications of jet ventilation include necrotizing tracheobronchitis, defoliating tumor cells, desiccation, hypothermia, inadequate ventilation with hypoxia and hypercapnia-especially in patients with low pulmonary and chest wall compliance-or jet flow not in alignment with the tracheobronchial tree $(10,11,18)$. Commercially available jet ventilators with built-in monitoring of airway pressure and familiarity of anesthesia providers with jet ventilation can ensure its safe usage (10).

Multiple catheters have been used for jet ventilation. Catheters need to be kink resistant with a small hole that allows jet flow. Laserjet and Hunsaker have been used extensively at our institution for tracheal surgery. There is no specially designed endobronchial jet catheter. Airway exchange catheters, bronchial blockers, and suction catheters have all been maneuvered into the trachea and main bronchus to allow for jet ventilation (19-23). Catheters can either be placed directly in the airway or placed through an ETT or laryngeal mask airway (LMA). The position of the jet catheter can be assisted and confirmed with a fiberoptic scope. When the airway is open, the surgeon can verify and adjust the location of the jet catheter or place the jet catheter in a retrograde fashion (24). It should be noted that if the jet catheter does not contain an anchoring device such as a balloon or basket, it can be challenging to maintain the catheter in a stable position during the surgery 
and prevent direct jet flow to mucosa that can cause injury.

\section{Nonintubated spontaneous breathing}

On October $16^{\text {th }}, 1846$, William Morton made the first public presentation of anesthetizing a patient for neck surgery at Massachusetts General Hospital (MGH) with inhalation of ether (25). Until the early twentieth century, most surgery was performed with patients spontaneously breathing to ensure the safety of patients, particularly those with a tenuous airway. The importance of spontaneous respiration was clearly recognized when tracheal surgery was conceived and implemented at MGH in its early days (14). For lower airway and intrathoracic surgery, the challenges were more daunting. Local anesthesia and regional anesthesia were adopted and published by Vischnevski and Ossipov in the 1950s to assist operations of major pulmonary surgery with patients spontaneously breathing $(26,27)$.

The advent of mechanical ventilation and betterdesigned ETT and DLT allowed most of thoracic surgery to be performed under general anesthesia with a secured airway in modern anesthesia. With the development of short-acting intravenous medication, local anesthetics, and minimally invasive procedures, there has been a renewed interest of carrying out thoracic surgery without intubation in the past decade $(28,29)$. Although the technique was initially targeted at pleural and peripheral pulmonary disease, it was quickly adopted to manage thoracic lesions across the whole spectrum $(28,30,31)$. It is natural to apply the technique in airway surgery since it accommodates for the optimal surgical field without impedance from airway devices.

Patient selection is critical in performing airway surgery with nonintubated techniques. Patients frequently will be sedated for comfort, minimizing coughing and mediastinum shift. Retention of carbon dioxide is a common phenomenon and could be as high as $80 \mathrm{mmHg}$ (32). Patients with expected difficult airway, type 2 respiratory failure, central hypoventilation syndrome, contralateral phrenic nerve palsy, high aspiration risk, and neurological disorders leading to high intracranial pressure or inability to cooperate, are not candidates for the techniques (33). In addition, need for contralateral lung isolation to protect the contralateral lung from contamination precludes usage of nonintubated techniques $(28,33,34)$. Relative contraindications include hemodynamic instability, obesity with body mass index (BMI) greater than 30, high airway secretions, coagulopathy, and extensive adhesions from prior surgery leading to surgical difficulty (33). Patients can be maintained as spontaneously breathing from the beginning or return to spontaneously breathing before the airway is open (35-37).

A variety of local, regional and neuraxial techniques have been adopted in nonintubated techniques to control pain, lower the dosage of sedatives, and decrease the risk of converting to intubation. Local infiltration, airway topicalization, cervical epidural, and superficial cervical plexus block have all been used for nonintubated cervical tracheal resection (38-40). Superficial cervical plexus block is sometimes chosen over cervical epidural to avoid the risk of high epidural or intrathecal block, hypotension, and bilateral phrenic nerve paralysis. For intrathoracic airway surgery such as carinal resection and reconstruction and sleeve lobectomy, regional techniques must address the pain from skin to parietal pleura stemming first from incision or video-assisted thoracoscopic surgery (VATS) port placement and then from visceral pleura and reflexes caused by manipulation and traction of intrathoracic structures. Intercostal nerve block, paravertebral nerve block, and epidural anesthesia have all been used to meet these goals (41). Preoperative lidocaine nebulization, stellar ganglion block or ipsilateral phrenic nerve block, and intrathoracic vagus nerve block by surgeons can all be utilized to minimize the cough reflex during operations $(28,32,34)$. Most patients require some amount of intravenous sedation in addition to the above techniques for comfort during the operation. Supraglottic airway devices can be deployed to help relieve airway obstruction, facilitate airway examination, supply oxygen, and provide positive-pressure ventilation before the airway is open $(32,40)$. Oxygen catheters can also be placed into the distal airway from surgical field if oxygen supplementation is necessary during the procedure. With correct patient selection and skillful surgical hands, the above techniques have enabled successful operations in many case series $(42,43)$.

The potential benefits of nonintubated technique are abundant. It avoids airway trauma from intubation and repeated airway manipulation and decreases the risk of mucosal damage from repeated airway instrumentation or jet flow. Absence of muscle relaxation and positive-pressure ventilation may decrease production of inflammatory factors and lower postoperative complications. Nonintubated techniques can minimize the risk of airway bleeding and tumor shedding (24). Better pain control, early ambulation, 
early chest tube removal, and decreased length of hospital stay have all been reported $(33,44-47)$. From the surgical standpoint, avoiding intubation holds the unique advantage of allowing surgeons to operate in an unobstructed fashion. It helps surgeons to identify lesions and the open field allows for a faster and simpler operation that decreases airway ischemia time, which may improve surgical outcomes (32).

Nevertheless, the challenges of nonintubated technique should not be neglected. The operations are performed without a secured airway, which requires expertise from both surgical and anesthesia teams. Close communication between the surgical and anesthesia teams is mandatory. Contingency airway plans must be in place such as oral intubation, cross-field ventilation, or jet ventilation if patients become hypoxemic, hemodynamic unstable, or have persistent coughing or excessive movement. Conversion may also be necessary in case of surgical complications like insufficient lung collapse, dense adhesions, and excessive bleeding $(33,34)$. The average conversion rate from nonintubated to intubated surgery is $2 \%$ to $3 \%$, with the range depending on the complexity of the surgery: minorintermediate procedures have a conversion rate of $1 \%$ while major procedures are around $10 \%(33,44)$. The complex nature of surgery, relatively small number of cases, and lack of prospective studies and outcome comparisons make it challenging to promote nonintubated spontaneously breathing techniques in airway surgery beyond dedicated high-volume centers at the current time (48).

\section{Extracorporeal membrane oxygenation (ECMO)}

Extracorporeal support for airway surgery dates back to the early 1960s. Cardiopulmonary bypass (CPB) was first used to support resection of an adenoid cystic carcinoma in the distal trachea (49-51). With improvement of membrane and centrifuge technology, ECMO emerged in the late 1970s to support respiratory failure, especially in the pediatric population. In 1992, Walker et al. first reported repair of congenital tracheal stenosis under ECMO (52). With its more favorable hematologic profile, ECMO has replaced $\mathrm{CPB}$ in most airway cases that require extracorporeal support.

Extracorporeal support is appealing for airway operations as it provides a clean surgical field with stable respiratory and hemodynamic parameters. Case report and case series have been published using ECMO for surgical resection and reconstruction of central airway neoplasms, endoscopic interventions for tumor debridement, stent placement, bronchopleural fistula, and tracheoesophageal fistula repair (53-59). While there is no consensus on the absolute indications for using ECMO in thoracic surgery, it should be considered in patients with critical intrathoracic airway stenosis, temporary complete airway occlusion, catastrophic trauma to the airway, or complex carinal reconstruction $(53,60)$. ECMO can also be used for intrathoracic airway surgery when patients lack pulmonary reserve or are unable tolerate OLV (61,62). Although veno-venous (VV) ECMO is predominantly deployed to provide respiratory support, veno-arterial (VA) ECMO should be utilized if there is hemodynamic instability secondary to airway occlusion, for operations that will involve retraction of the heart, mediastinum, and large vessels, or for patients in whom the heart will obscure the surgical field $(48,61)$.

Airway surgery has been done with ECMO support safely. Lang et al. reported using VA ECMO on 10 patients for complex tracheobronchial surgery without neurologic complications (59). Willms et al. applied VV- or VA-ECMO on 12 patients with acute tracheal obstruction and all were successfully discharged home (56). However, the decision to choose ECMO should not be made lightly. Inherent risks of ECMO such as bleeding, vascular structure injury, and ischemia may occur and require closely monitoring. $\mathrm{VV}$-ECMO can have problems of recirculation, mixture of oxygenated and de-oxygenated blood, chatter, and suck-down. Adequate intravascular volume is required to maintain oxygen saturation and venous drainage, which can result in volume overload in thoracic patients (63). Left ventricular distension and pulmonary edema can occur in VA-ECMO. Harlequin syndrome requires special attention in VA-ECMO patients for potential cerebral hypoxia (64). Not infrequently, patients will need ECMO support or mechanical ventilation support after surgery that may jeopardize anastomotic viability (54). Given these risks, in general, selection of ECMO as the primary method of airway management should be executed under extreme caution.

\section{Anesthesia for cervical tracheal resection and reconstruction}

Proximal tracheal resection and reconstruction is the one of most frequently performed airway reconstructive operations. Post-intubation tracheal stenosis used to account for most cases, with other causes including primary tracheal or metastatic tracheal tumors as well as idiopathic tracheal stenosis (65). Better designed ETTs with low cuff pressure and meticulous care with mechanical ventilation have 


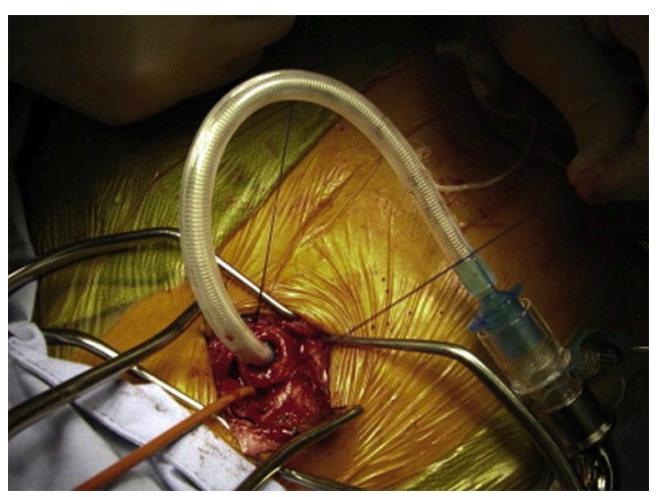

Figure 1 The red rubber catheter is stitched to the orotracheal tube, which has been withdrawn out of the field, leaving the catheter emerging from the proximal trachea. A sterile tube is placed within the distal tracheal stump while the surgeon performs the tracheal reconstruction. Reprinted with permission from Chitilian HV, Bao X, Mathisen DJ, et al. Anesthesia for Airway Surgery. Thorac Surg Clin 2018;28:249-55.

significantly decreased the number of patients with critical tracheal stenosis. Post-tracheostomy injury is currently responsible for the most cases of airway stenosis (66). The COVID-19 pandemic created an unprecedented surge of critical ill patients requiring mechanical ventilation and tracheostomy and some may likely require tracheal resection and reconstruction in the near future.

Meticulous preoperative assessment of the patient undergoing tracheal resection and reconstruction is critical to its success. As with any case, cardiopulmonary reserve must be evaluated and comorbidities optimized so patients can tolerate this moderate-risk surgery. Patients should be assessed for mental status, cervical flexibility, and intracranial pressure. Upper airway obstruction and chest wall compliance should be determined before the operation. Dyspnea with exertion, dyspnea at rest, and inspiratory stridor can provide a rough estimate of disease severity.

Detailed information about the tracheal lesion is mandatory. High-resolution neck and chest CT determines location, size, and extent of tracheal lesion, which are essential for selecting the appropriate airway instrument. Pulmonary function tests are not necessary unless there is concern for intrathoracic disease process. Fiberoptic or rigid bronchoscopy exams are mandatory because they not only provide accurate information regarding the tracheal lesion but also allow for serial dilation to accommodate the appropriately sized ETT.

Premedication should be judicious depending on the severity of the patient's airway disease. Respiratory depression must be avoided for any patient with a severely stenotic airway and symptomatic disease. Standard monitors and single peripheral intravenous access suffice for most cases. Continuous arterial monitoring depends upon patient comorbidities and specific airway management needs. Inhalational inductions were traditionally advocated for patients with severe tracheal stenosis due to concern of losing the airway. At our center, intravenous induction is routinely utilized for patients with tracheal stenosis and positive-pressure mask ventilation was achievable in all patients even with airways as narrow as 1 to $2 \mathrm{~mm}$. Our findings are consistent with that of other groups that even with tracheal diameters between $10 \%$ to $40 \%$ of normal, there were no reports of difficulties with positive-pressure ventilation through an LMA and peak pressures did not exceed 15 to $18 \mathrm{cmH}_{2} \mathrm{O}$ despite severe airway stenosis $(24,67,68)$. For all cases with critical airways, surgeons should be present in the operating room prior to induction of anesthesia with rigid bronchoscopes of different sizes available in case of obstruction or need for surgical airway.

Total intravenous anesthesia (TIVA) is standard practice to maintain a stable level of anesthesia during operations. Cross-field ventilation is the most frequently utilized airway management with detailed description in our prior publications $(69,70)$. In brief, patients are intubated with the appropriately sized ETT. Immediately prior to opening the airway, the ETT is cautiously retracted above the stenosis. Once the airway is open, a red rubber catheter is sutured to the distal end of the ETT and a sterile flexible ETT is inserted into the distal trachea as shown (Figure 1). Crossfield ventilation and intermittent apnea allow the operation to proceed (Figure 2). Before tying down the final suture for complete anastomosis, the oral ETT is advanced to the distal tracheal under the guidance of the red rubber catheter and direct visualization.

Jet ventilation has been adopted in tracheal resection to eliminate the need for intermittent apnea. LMA can be invaluable to facilitate jet ventilation. Placement of an LMA allows for detailed airway examination and dilation if necessary. A LaserJet catheter can fit through an LMA and placed across the stenotic region under bronchoscopic guidance (Figure 3). Conventional positive-pressure ventilation is accomplished through the LMA until the airway is open, at which point jet ventilation can commence. The jet catheter can also be placed retrograde through the open airway if unable to be placed anterogradely (24). Reconstruction can be carried out around the jet catheter 


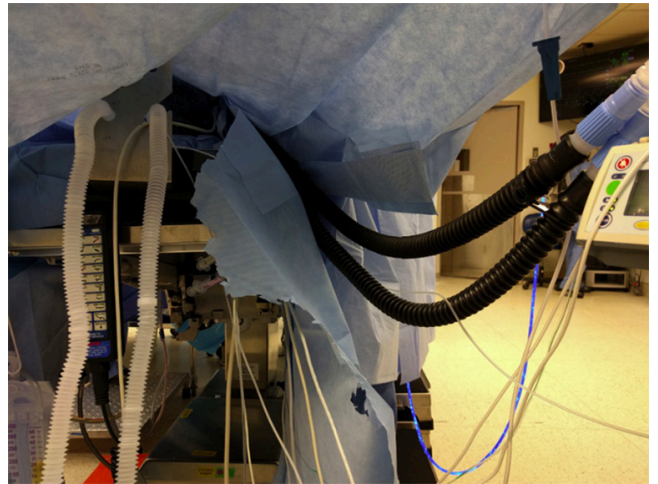

Figure 2 The black circuit tubing is connected to the sterile tube and establishes cross-field ventilation. The clear circuit tubing to the left remains connected to the orotracheal tube. The surgical team often places a new up-drape after the cross-field circuit is passed to the anesthesiologist, and the old sterile up-drape is dropped and cut to improve access to the patient's face. Reprinted with permission from Chitilian HV, Bao X, Mathisen DJ, et al. Anesthesia for Airway Surgery. Thorac Surg Clin 2018;28:249-55.

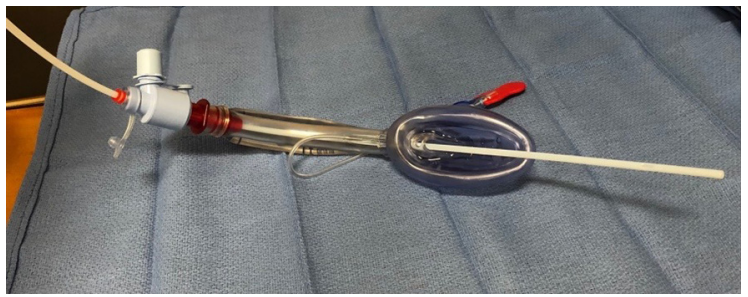

Figure 3 A LaserJet jet catheter is placed through a laryngeal mask airway (LMA) via a bronchoscope adapter to provide jet ventilation. A side channel provides a sampling port that allows for end-tidal carbon dioxide detection during the surgery. The primary anesthesia circuit remains attached to the LMA via the bronchoscope adapter.

without requiring apneic periods (Figure 4). Attention must be paid to jet ventilation complications as above.

\section{Anesthesia management for carinal resection and reconstruction}

Carinal resection has been described as one of the most complex thoracic surgeries. The operation involves sophisticated resection and reconstruction of trachea and bronchi. The procedure is indicated for non-small cell lung cancer, low-grade malignant tumors, and benign tumors involving the distal trachea, carina, and/or proximal

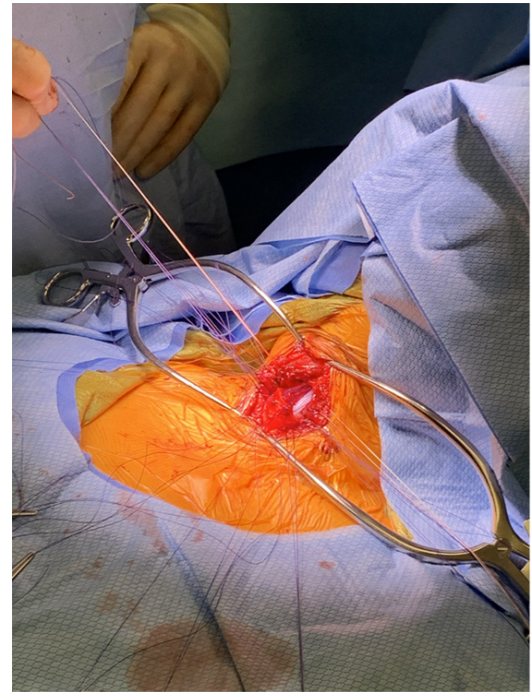

Figure 4 The jet catheter passes into the surgical field with minimal obstruction, allowing the surgeon to operate around the catheter and avoiding periods of apnea.

mainstem bronchi. Contraindications include the need to resect greater than $4 \mathrm{~cm}$ of the distal trachea or greater than $1.5 \mathrm{~cm}$ of proximal bronchi (71). Traditionally, the procedure is undertaken with a thoracotomy, with mortality of $2.2 \%$ to $7.1 \%$ and morbidity of $39 \%$ to $50 \%$ (72).

The specific procedure is based upon the location of the tumor and surgical experience. In a recent case series from MGH of 45 cases over the course of twenty years, $47 \%$ of the cases were carinal resection with the construction of a neocarina, $31 \%$ were right carinal pneumonectomies, $20 \%$ were left carinal pneumonectomies, and $2 \%$ were carinal resections with lobar resections (73). The surgical approach also varies depending on tumor location, with patients undergoing right thoracotomy (58\%), median sternotomy (33\%), left thoracotomy (4\%), and median sternotomy with right thoracotomy (4\%). Smaller case series have described a successful right-sided VATS approach to the procedure $(74,75)$. Investigations are ongoing to determine the optimal surgical technique. A case control study of 18 patients undergoing VATS tracheal or carinal resection with spontaneous ventilation (14 tracheal, 4 carinal) compared to 14 historic controls (12 tracheal, 2 carinal) who underwent the procedure through a right thoracotomy under general anesthesia with mechanical ventilation reported a shorter median operative time in the VATS group with comparable morbidity and outcomes (75). Potential confounders were that the VATS group included only American Society of Anesthesiologists 
class I or II patients with BMI less than 25 (75). There have also been reports of successful single-port VATS carinal resections (76,77).

Anesthetic management begins with a thorough understanding of the surgical plan. Patients are maintained under a TIVA to ensure consistent depth of anesthesia (69). For patients undergoing a thoracotomy, epidural analgesia is used for postoperative pain control. The specific approach to airway management is determined in discussion with the surgeon and based on airway anatomy and tumor morphology. Endobronchial intubation of the contralateral mainstem bronchus with a long, small caliber tube is used most often to manage the airway. Depending on the surgical plan, the tube can be left in or withdrawn with initiation of cross-field ventilation once the surgeon enters the airway; this approach has a longstanding record of safety and efficacy $(71,73)$. The airway can also be managed with an ETT placed above the lesion and a bronchial blocker inserted distal to the lesion. Univent tubes can also be a versatile tool due to their unique design.

Multiple jet ventilation methods have been designed for carinal surgery. The jet catheter can be placed through an ETT or cross-field on its own. Double jet catheters can be placed at the right and left bronchi for HFJV to maintain good oxygenation and normocapnia for patients sensitive to carbon dioxide retention $(78,79)$. Jet ventilation can also be accomplished through a bronchial blocker $(19,22)$.

Recently, cases series have been published describing carinal resection performed by VATS in nonintubated, spontaneously ventilating patients $(32,74,75)$. Patients were kept spontaneous breathing after regional block and LMA insertion. The absence of an airway device in the field allows the surgeon to operate in an easier fashion. As mentioned above, appropriate patient selection is critical in this approach. Further study is needed to determine whether it leads to improved outcomes and fewer complications $(32,75)$.

Mechanical circulatory support should be considered for patients with tumors extending into central structures or near major vessels who may require significant cardiac manipulation intraoperatively or for whom there is concern for the ability to perform OLV (73).

\section{Conclusions}

Over half a century has passed since Grillo first published the successful performance of tracheal reconstruction at MGH (80). With limited resources at hand, the unique challenges of maintaining the airway and delivering anesthesia were met with anesthetic and surgical ingenuity. Spontaneous breathing of halothane and establishing crossfield circuits ensured smooth operations with favorable outcomes in the early days (14). With better understanding of airway anatomy, improved anastomotic skill, and innovative instruments, airway surgery has made leaps over the past decades. Multiple surgical approaches have been developed to manage lesions from the level of the trachea to the bronchus. In response, numerous anesthetic and airway management methods have evolved. An anesthesiologist's full understanding of different airway management techniques with special attention to the location of the airway lesion can facilitate orchestration of safe and successful airway surgery.

At the same time, there are limited prospective studies in comparing the outcomes of different approaches. Publications in the area are mostly case reports or small case series as the relatively low number of cases means it is challenging to carry out randomized trials in the area. It took us over a decade to refine the details for cross-field ventilation, We can only expect to continuously practice, study, and analyze new approaches to airway operations. Attention should be paid not only to performing operations safely, but also to minimizing surgical complications and improving patient recovery. Research on specific biomarkers for stress response and inflammation deserves attention as they may serve as surrogates of patient recovery. Modeling of shear stress on the airway wall by different ventilation techniques could help justify selection of airway methods. Future research should also focus on researching techniques and pharmacological agents that improve wound healing and at the same time minimize risk of re-stenosis. Collaboration among high-volume centers can pave the way for hypothesis testing and guideline development. In the end, as medical professionals, we should never be content with the status quo, but must continue exploring.

\section{Acknowledgments}

Funding: None.

\section{Footnote}

Reporting Checklist: The authors have completed the Narrative Review reporting checklist. Available at https:// dx.doi.org/10.21037/tlcr-21-917

Conflicts of Interest: All authors have completed the ICMJE 
uniform disclosure form (available at https://dx.doi. org/10.21037/tlcr-21-917). The authors have no conflicts of interest to declare.

Ethical Statement: The authors are accountable for all aspects of the work in ensuring that questions related to the accuracy or integrity of any part of the work are appropriately investigated and resolved.

Open Access Statement: This is an Open Access article distributed in accordance with the Creative Commons Attribution-NonCommercial-NoDerivs 4.0 International License (CC BY-NC-ND 4.0), which permits the noncommercial replication and distribution of the article with the strict proviso that no changes or edits are made and the original work is properly cited (including links to both the formal publication through the relevant DOI and the license). See: https://creativecommons.org/ licenses/by-nc-nd/4.0/.

\section{References}

1. Carter MG, Strieder JW. Resection of the trachea and bronchi: an experimental study. J Thorac Surg 1950;20:613-27.

2. Juvenelle A, Citret C. Transplantation of the main bronchus and resection of the tracheal bifurcation: experimental study in the dog. J Chir (Paris) 1951;67:666-88.

3. Baumann J. Anesthesia in tracheal surgery. Poumon Coeur 1960;16:19-32.

4. Grillo HC. Circumferential resection and reconstruction of the mediastinal and cervical trachea. Ann Surg 1965;162:374-88.

5. Schieren M, Böhmer A, Dusse F, et al. New Approaches to Airway Management in Tracheal Resections-A Systematic Review and Meta-analysis. J Cardiothorac Vasc Anesth 2017;31:1351-8.

6. Tapias LF, Mathisen DJ. Prevention and management of complications following tracheal resections-lessons learned at the Massachusetts General Hospital. Ann Cardiothorac Surg 2018;7:237-43.

7. Bernoulli D. Hydrodynamica, sive de viribus et motibus fluidorum commentarii. Strassburg (DE): Johannis Reinholdi Dulseckeri, 1738.

8. Sanders R. Two ventilating attachments for bronchoscopes. Med J 1967;39:170.

9. Klain M, Smith RB. High frequency percutaneous transtracheal jet ventilation. Crit Care Med 1977;5:280-7.

10. Biro P. Jet ventilation for surgical interventions in the upper airway. Anesthesiol Clin 2010;28:397-409.

11. Evans E, Biro P, Bedforth N. Jet ventilation. Contin Educ Anaesth Crit Care Pain 2007;7:2-5.

12. Slutsky AS, Drazen JM. Ventilation with small tidal volumes. N Engl J Med 2002;347:630-1.

13. Ihra G, Gockner G, Kashanipour A, et al. Highfrequency jet ventilation in European and North American institutions: developments and clinical practice. Eur J Anaesthesiol 2000;17:418-30.

14. Geffin B, Bland J, Grillo HC. Anesthetic management of tracheal resection and reconstruction. Anesth Analg 1969;48:884-90.

15. Ihra GC, Heid A, Pernerstorfer T. Airway stenosis-related increase of pulmonary pressure during high-frequency jet ventilation depends on injector's position. Anesth Analg 2009;109:461-5.

16. Buczkowski PW, Fombon FN, Lin ES, et al. Air entrainment during high-frequency jet ventilation in a model of upper tracheal stenosis. Br J Anaesth 2007;99:891-7.

17. Pertile J, Smith B, Mellenthin M, et al. Jet flow rate and needle position govern distal airway pressures during low-frequency jet ventilation. Laryngoscope Investig Otolaryngol 2021;6:244-51.

18. Cook TM, Alexander R. Major complications during anaesthesia for elective laryngeal surgery in the UK: a national survey of the use of high-pressure source ventilation. Br J Anaesth 2008;101:266-72.

19. Chin JH, Lee EH, Choi DK, et al. High frequency jet ventilation of one lung using a bronchial blocker of Univent during carinal resection. J Korean Med Sci 2010;25:1083-5.

20. Ng JM. Hypoxemia during one-lung ventilation: jet ventilation of the middle and lower lobes during right upper lobe sleeve resection. Anesth Analg 2005;101:1554-5.

21. Luyet C, Boudah R, McCartney CJ, et al. Lowfrequency jet ventilation through a bronchial blocker for tracheal repair after a rare complication of percutaneous dilatational tracheostomy. J Cardiothorac Vasc Anesth 2013;27:108-10.

22. Williams H, Gothard J. Jet ventilation via a Univent tube for sleeve pneumonectomy. Eur J Anaesthesiol 2001;18:407-9.

23. Hautmann H, Gamarra F, Henke M, et al. High frequency 
jet ventilation in interventional fiberoptic bronchoscopy. Anesth Analg 2000;90:1436-40.

24. Schieren M, Egyed E, Hartmann B, et al. Airway Management by Laryngeal Mask Airways for Cervical Tracheal Resection and Reconstruction: A Single-Center Retrospective Analysis. Anesth Analg 2018;126:1257-61.

25. Viets HR. The earliest printed references in newspapers and journals to the first public demonstation of ether anesthesia in 1846. J Hist Med Allied Sci 1949;4:149-69.

26. Vischnevski AA. Local anesthesia in thoracic surgery: lungs, heart and esophagus. Minerva Anestesiol 1954;20:432-5.

27. Ossipov BK. Local anesthesia in thoracic surgery: 20 years experience with 3265 cases. Anesth Analg 1960;39:327-32.

28. Gonzalez-Rivas D, Bonome C, Fieira E, et al. Nonintubated video-assisted thoracoscopic lung resections: the future of thoracic surgery? Eur J Cardiothorac Surg 2016;49:721-31.

29. Pompeo E. State of the art and perspectives in nonintubated thoracic surgery. Ann Transl Med 2014;2:106.

30. Wong MKH, Sit AKY, Au TWK. Minimally invasive thoracic surgery: beyond surgical access. J Thorac Dis 2018;10:S1884-91.

31. Akopov A, Kovalev M. Nonintubated Tracheal Surgery. Thorac Surg Clin 2020;30:91-9.

32. Liang H, Gonzalez-Rivas D, Zhou Y, et al. Nonintubated Anesthesia for Tracheal/Carinal Resection and Reconstruction. Thorac Surg Clin 2020;30:83-90.

33. Irons JF, Martinez G. Anaesthetic considerations for nonintubated thoracic surgery. J Vis Surg 2016;2:61.

34. Kiss G. Technical Issues and Patient Safety in Nonintubated Thoracic Anesthesia. Thorac Surg Clin 2020;30:1-13.

35. Liu X, Jiang R, Xiao J, et al. Anesthesia airway management for tracheal resection and reconstruction: a single-center case series. Ann Palliat Med 2021;10:3354-63.

36. Al-Abdullatief M, Wahood A, Al-Shirawi N, et al. Awake anaesthesia for major thoracic surgical procedures: an observational study. Eur J Cardiothorac Surg 2007;32:346-50.

37. Zhang XX, Song CT, Gao Z, et al. A comparison of nonintubated video-assisted thoracic surgery with spontaneous ventilation and intubated video-assisted thoracic surgery: a meta-analysis based on 14 randomized controlled trials. J Thorac Dis 2021;13:1624-40.

38. Macchiarini P, Rovira I, Ferrarello S. Awake upper airway surgery. Ann Thorac Surg 2010;89:387-90; discussion 390-1.

39. Liu J, Li S, Shen J, et al. Non-intubated resection and reconstruction of trachea for the treatment of a mass in the upper trachea. J Thorac Dis 2016;8:594-9.

40. Zhou Y, Liu H, Wu X, et al. Spontaneous breathing anesthesia for cervical tracheal resection and reconstruction. J Thorac Dis 2019;11:5336-42.

41. Yang JT, Hung MH, Chen JS, et al. Anesthetic consideration for nonintubated VATS. J Thorac Dis 2014;6:10-3.

42. Shao W, Phan K, Guo X, et al. Non-intubated complete thoracoscopic bronchial sleeve resection for central lung cancer. J Thorac Dis 2014;6:1485-8.

43. Furák J, Szabó Z, Tánczos T, et al. Conversion method to manage surgical difficulties in non-intubated uniportal video-assisted thoracic surgery for major lung resection: simple thoracotomy without intubation. J Thorac Dis 2020;12:2061-9.

44. Tacconi F, Pompeo E. Non-intubated video-assisted thoracic surgery: where does evidence stand? J Thorac Dis 2016;8:S364-75.

45. Liu J, Cui F, Li S, et al. Nonintubated video-assisted thoracoscopic surgery under epidural anesthesia compared with conventional anesthetic option: a randomized control study. Surg Innov 2015;22:123-30.

46. Xue W, Duan G, Zhang X, et al. Comparison of nonintubated and intubated video-assisted thoracoscopic surgeries of major pulmonary resections for lung cancer-a meta-analysis. World J Surg Oncol 2021;19:87.

47. Zhang K, Chen HG, Wu WB, et al. Non-intubated videoassisted thoracoscopic surgery vs. intubated video-assisted thoracoscopic surgery for thoracic disease: a systematic review and meta-analysis of 1,684 cases. J Thorac Dis 2019;11:3556-68.

48. Schweiger T, Klepetko W, Hoetzenecker K. Awake minimal invasive carinal resection-tightrope walking in thoracic surgery?. J Thorac Dis 2017;9:3667-9.

49. Woods FM, Neptune WB, Palatchi A. Resection of the carina and main-stem bronchi with the use of extracorporeal circulation. N Engl J Med 1961;264:492-4.

50. Adkins PC, Izawa EM. Resection of tracheal cylindroma using cardiopulmonary bypass. Arch Surg 1964;88:405-9.

51. Nissen R. Extracorporeal circulation for prolonged (30 minutes) respiratory interruption in surgery of tracheal tumors in the area of the bifurcation. Schweiz Med 
Wochenschr 1961;91:957-64.

52. Walker LK, Wetzel RC, Haller JA Jr. Extracorporeal membrane oxygenation for perioperative support during congenital tracheal stenosis repair. Anesth Analg 1992;75:825-9.

53. Hoetzenecker K, Klepetko W, Keshavjee S, et al. Extracorporeal support in airway surgery. J Thorac Dis 2017;9:2108-17.

54. Pu H, Lei Y, Yuan D, et al. Tracheal Reconstruction Surgery Supported by Extracorporeal Membrane Oxygenation for Patients with Traumatic PostTracheotomy Tracheal Stenosis. Ann Thorac Cardiovasc Surg 2020;26:327-31.

55. Sanchez-Lorente D, Iglesias M, Rodríguez A, et al. The pumpless extracorporeal lung membrane provides complete respiratory support during complex airway reconstructions without inducing cellular trauma or a coagulatory and inflammatory response. J Thorac Cardiovasc Surg 2012;144:425-30.

56. Willms DC, Mendez R, Norman V, et al. Emergency bedside extracorporeal membrane oxygenation for rescue of acute tracheal obstruction. Respir Care 2012;57:646-9.

57. Kim SH, Song S, Kim YD, et al. Outcomes of Extracorporeal Life Support During Surgery for the Critical Airway Stenosis. ASAIO J 2017;63:99-103.

58. Hong Y, Jo KW, Lyu J, et al. Use of venovenous extracorporeal membrane oxygenation in central airway obstruction to facilitate interventions leading to definitive airway security. J Crit Care 2013;28:669-74.

59. Lang G, Ghanim B, Hötzenecker K, et al. Extracorporeal membrane oxygenation support for complex tracheobronchial procedures $†$. Eur J Cardiothorac Surg 2015;47:250-5; discussion 256.

60. Abdelmalak BB, Doyle DJ. Recent trends in airway management. F1000Res 2020;9:eF1000 Faculty Rev-355.

61. Weder W, Inci I. Carinal resection and sleeve pneumonectomy. J Thorac Dis 2016;8:S882-8.

62. Reeb J, Olland A, Massard G, et al. Extracorporeal life support in thoracic surgery. Eur J Cardiothorac Surg 2018;53:489-94.

63. Tonna JE, Abrams D, Brodie D, et al. Management of Adult Patients Supported with Venovenous Extracorporeal Membrane Oxygenation (VV ECMO): Guideline from the Extracorporeal Life Support Organization (ELSO). ASAIO J 2021;67:601-10.

64. Rao P, Khalpey Z, Smith R, et al. Venoarterial Extracorporeal Membrane Oxygenation for Cardiogenic Shock and Cardiac Arrest. Circ Heart Fail 2018;11:e004905.

65. Grillo HC, Zannini P, Michelassi F. Complications of tracheal reconstruction. Incidence, treatment, and prevention. J Thorac Cardiovasc Surg 1986;91:322-8.

66. Kim SS, Khalpey Z, Hsu C, et al. Changes in Tracheostomy- and Intubation-Related Tracheal Stenosis: Implications for Surgery. Ann Thorac Surg 2017;104:964-70.

67. Biro P, Hegi TR, Weder W, et al. Laryngeal mask airway and high-frequency jet ventilation for the resection of a high-grade upper tracheal stenosis. J Clin Anesth 2001;13:141-3.

68. Adelsmayr E, Keller C, Erd G, et al. The laryngeal mask and high-frequency jet ventilation for resection of high tracheal stenosis. Anesth Analg 1998;86:907-8.

69. Chitilian HV, Bao X, Mathisen DJ, et al. Anesthesia for Airway Surgery. Thorac Surg Clin 2018;28:249-55.

70. Hobai IA, Chhangani SV, Alfille PH. Anesthesia for tracheal resection and reconstruction. Anesthesiol Clin 2012;30:709-30.

71. Tapias L, Lanuti M. Carinal resections. Ann Cardiothorac Surg 2018;7:309-13.

72. Guido-Guerrero W, Bolaños-Cubillo A, GonzálezRivas D. Single-port video-assisted thoracic surgery (VATS)-advanced procedures \& update. J Thorac Dis 2018;10:S1652-S1661.

73. Costantino CL, Geller AD, Wright CD, et al. Carinal surgery: A single-institution experience spanning 2 decades. J Thorac Cardiovasc Surg 2019;157:2073-2083.e1.

74. Li J, Wang W, Jiang L, et al. Video-Assisted Thoracic Surgery Resection and Reconstruction of Carina and Trachea for Malignant or Benign Disease in 12 Patients: Three Centers' Experience in China. Ann Thorac Surg 2016;102:295-303.

75. Jiang L, Liu J, Gonzalez-Rivas D, et al. Thoracoscopic surgery for tracheal and carinal resection and reconstruction under spontaneous ventilation. J Thorac Cardiovasc Surg 2018;155:2746-54.

76. Gonzalez-Rivas D, Yang Y, Stupnik T, et al. Uniportal video-assisted thoracoscopic bronchovascular, tracheal and carinal sleeve resections†. Eur J Cardiothorac Surg 2016;49 Suppl 1:i6-i16.

77. Hung WH, Chen HC, Huang CL, et al. Thoracoscopic Tracheal Resection and Reconstruction With SingleIncision Method. Ann Thorac Surg 2018;106:e45-7.

78. Perera ER, Vidic DM, Zivot J. Carinal resection with two high-frequency jet ventilation delivery systems. Can J Anaesth 1993;40:59-63. 
79. Crinquette V, Wurtz A, Leroy S, et al. Resection and reconstruction of the carina with separate two-lung highfrequency jet ventilation. Ann Chir 1989;43:673-6.

Cite this article as: Li LT, Chitilian HV, Alfille PH, Bao X. Airway management and anesthesia for airway surgery: a narrative review. Transl Lung Cancer Res 2021;10(12):46314642. doi: 10.21037/tlcr-21-917
80. Grillo HC. The management of tracheal stenosis following assisted respiration. J Thorac Cardiovasc Surg 1969;57:52-71. 